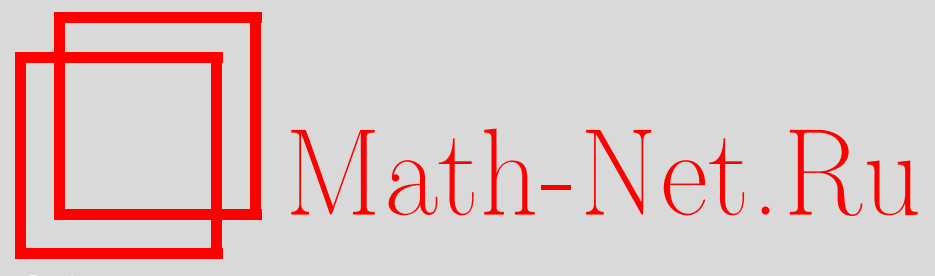

Н. С. Иманбаев, Задача о собственных значениях дифференциального оператора Коши-Римана c нелокальными краевыми условиями, Вестн. Сам. гос. техн. ун-та. Сер. Физ.-мат. науки, 2014, выпуск 1(), 25-36

DOI: https://doi.org/10.14498/vsgtu1264

Использование Общероссийского математического портала MathNet.Ru подразумевает, что вы прочитали и согласны с пользовательским соглашением

http://www.mathnet.ru/rus/agreement

Параметры загрузки:

IP: 35.173 .219 .12

26 апреля 2023 г., 07:04:18

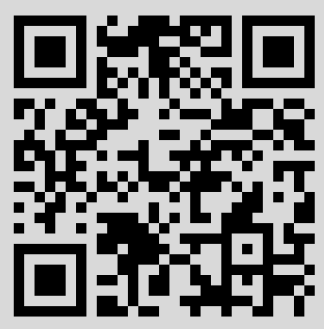


Вестн. Сам. гос. техн. ун-та. Сер. Физ.-мат. науки. 2014. № 1 (34). С. $25-36$

УДК 517.984.5

\title{
ЗАДАЧА О СОБСТВЕННЫХ ЗНАЧЕНИЯХ ДИФФЕРЕНЦИАЛЬНОГО ОПЕРАТОРА КОШИ-РИМАНА С НЕЛОКАЛЬНЫМИ КРАЕВЫМИ УСЛОВИЯМИ
}

\section{Н. С. Иманбаев}

Международный казахско-турецкий университет им. Х. А. Ясави, Казахстан, 487010, Туркестан, пл. Есим-Хана, 2.

\begin{abstract}
Рассматривается редуцированная спектральная задача для оператора КошиРимана с нелокальными краевыми условиями $к$ линейному интегральному уравнению Фредголъма второго рода с непрерывным ядром. Соответствующий детерминант Фредгольма определён при всех спектралъных параметрах $\lambda$, кроме $\lambda \neq 2, \operatorname{Re} \lambda \neq 1$. Нахождение нулей определителя Фредгольма, записанного в такой борме, неэфбективно, поскольку он не является иелой бункиией от спектрального параметра, а его главная часть не выделена. Исследована структура ядра оператора. Для приближённого решения интегралъного уравнения применены результаты работ И. Акбергенова, где даны оценки абсолютной величины разности между точным и приближенным решениями интегралъного уравнения. Охарактеризованы спектралъные параметры, при которых неоднородная краевая задача со смещением для уравнений Коши-Римана всюду разрешима в классе непрерьвных функиий на единичном круге. Показана явная конструкиия, аппроксимирующая решение неоднородной краевой задачи.
\end{abstract}

Ключевые слова: оператор Коши-Римана, пространство непрерывных функиий, фредгольмовость, резольвентное множество, задача со смещением, ядро, детерминант Фредголъма.

В спектральной теории дифференциальных операторов в последние годы все большее внимание уделяется задачам для уравнений с частными производными, в которых краевые условия представляют собой соотношения между значениями искомых функций, вычисленными в различных точках, лежащих на границе или внутри рассматриваемой области.

Этот интерес объясняется как теоретической значимостью получаемых результатов, так и возможностями важных приложений. Подобные граничные условия возникают при математическом моделировании задач газовой динамики, теории плазмы, теплопроводности, излучения лазера, прогнозирования почвенной влаги, при изучении процессов размножения клеток, бактерий и т.п. В некоторых случаях (физика сверхпроводников, радиационный перенос, процессы распространения загрязнения в воде биосферы, демография, популяционная генетика и другие биологические проблемы) граничные условия имеют интегральную форму.

Примеры указанных краевых условий, возникающих в теплопроводности,

ISSN: 2310-7081 (online), 1991-8615 (print); doi: http://dx.doi.org/10.14498/vsgtu1264 (C) 2014 Самарский государственный технический университет.

Образец цитирования: Н. С. Им ан ба ев, "Задача о собственных значениях дифференциального оператора Коши-Римана с нелокальными краевыми условиями" // Вестн. Сам. гос. техн. ун-та. Сер. Физ.-мат. науки, 2014. № 1 (34). С. 25-36. doi: 10.14498/vsgtu1264. Сведения об авторе: Нурлан Сайрамович Иманбаев (к.ф.-м.н., доц.), профессор, каф. математики.

E-mail address: imanbaevnur@mail.ru 
были сформулированы ещё В. А. Стекловым [1], а в газовой динамикеФ. И. Франклем [2]. А. М. Нахушевым [3,5] были поставлены и изучены сразу несколько задач данного типа, а для их названия предложен термин «задачи со смещением». В статье А. В. Бицадзе и А. А. Самарского [4] впервые была исследована задача «со смещением внутрь области». Содержание последних публикаций привело к осознанию качественной новизны краевых задач со смещениями для теории дифференциальных уравнений в частных производных. В последних публикациях [6] для уравнений Бицадзе-Лыкова поставлена задача со смещением с операторами Кобера-Эрдейи и М. Сайго в краевом условии, а также исследованы вопросы единственности и неединственности решения задачи при различных функциях и значениях констант, входящих в краевые условия. В работах $[5,7]$ исследовано состояние краевых задач со смещением для основных типов уравнений в частных производных. Краевая задача со смещением для уравнения Карлемана-Векуа с сингулярной точкой изучалась в работе [8]. Обилие публикаций, где изучаются всё более общие ситуации, производит иногда впечатление, что теория краевых задач «со смещением» уже завершена. Однако здесь имеется ряд менее изученных, но важных вопросов, в частности, задача о собственных значениях или об их аналитическом описании с помощью, например, асимптотических разложений. Применяемые в настоящее время методы функционального анализа и метод сведения к модельным уравнениям путём интегральных преобразований, с одной стороны, недостаточны для того, чтобы получить такую детальную информацию. С другой стороны, вряд ли можно надеяться получить, например, решение задачи на собственные значения для дифференциальных уравнений с частными производными в столь же явном виде, как это было сделано в аналогичной ситуации для обыкновенных дифференциальных уравнений.

Общность перечисленных выше вопросов для уравнений с частными производными вынуждает в дальнейшем наложить на изучаемые операторы ряд весьма жёстких ограничений. Выяснение правильных постановок задач и исследование специфических свойств решений для «неклассических» уравнений удобно начинать с рассмотрения идеализированных моделей, например, уравнений с постоянными коэффициентами.

В связи с вышеизложенным целями настоящей работы являются:

1) исследование спектральной задачи для оператора Коши-Римана с нелокальными краевыми условиями

$$
\begin{gathered}
\frac{\partial \omega}{\partial \bar{z}}=\lambda \omega+f(z), \quad|z|<1 \\
\operatorname{Re} \omega(z)=\operatorname{Re}\left(\frac{1}{2 \pi i} \oint_{|t|=1} \frac{\lambda \omega(t)+f(t)}{t-z} d t\right), \quad|z|=1 \\
\operatorname{Im} \omega(0)=\operatorname{Im}\left(\frac{1}{2 \pi i} \oint_{|t|=1} \frac{\lambda \omega(t)+f(t)}{t} d t\right)
\end{gathered}
$$

где $\lambda$ - комплексное число (спектральный параметр);

2) приближенное решение краевой задачи (1)-(3).

Описание общих регулярных краевых задач для дифференциального выражения Коши-Римана разработано Дж. Ф. Нейманом, М. И. Вишиком, 
А. А. Дезиным, М. Отелбаевым. Приведённая задача носит нелокальный характер, и подобные задачи для операции Коши-Римана описаны М. Отелбаевым, А. Н. Шыныбековым [9].

$\mathrm{C}$ другой точки зрения вопросы разрешимости и поведения решения краевой задачи для обобщенного уравнения Коши-Римана глубоко изучались в работах [10-12]. Краевые задачи для обобщенной системы Коши-Римана с негладкими коэффициентами исследовались в работах $[13,14]$.

В функциональном пространстве $C(|z| \leqslant 1)$ рассмотрим оператор $K$, порождаемый дифференциальной операцией Коши-Римана

$$
K \omega(z)=\frac{\partial \omega(z)}{\partial \bar{z}},
$$

где

$$
z=x+i y, \quad \bar{z}=x-i y, \quad \frac{\partial}{\partial \bar{z}}=\frac{1}{2} \frac{\partial}{\partial x}+i \frac{\partial}{\partial y}
$$

на множестве

$$
D(K) \subset\left\{\omega(z) \in C(|z| \leqslant 1), \frac{\partial \omega}{\partial \bar{z}} \in C(|z|<1)\right\} .
$$

Считаем, что оператор $K$ имеет непустое резольвентное множество $\rho(K)$. Не умаляя общности, предполагаем

$$
0 \in \rho(K),
$$

т. е. существует ограниченный оператор $K^{-1}$. В работе [9] описано множество операторов $\{K\}$ со свойством (4).

Наиболее глубокие результаты при исследовании спектра эллиптических операторов приведены в работе [15]. В общем случае спектр эллиптического оператора существенно определяется спектральными свойствами граничного оператора. Однако выяснение зависимости спектра оператора Коши-Римана в исходных терминах граничных условий представляет актуальную (и нерешённую) проблему. В статье [16] в ограниченной области с достаточно гладкой границей рассматриваются основные краевые задачи для полулинейных уравнений эллиптического типа со спектральным параметром и разрывной нелинейностью. В работе [17] задача (1)-(3) на собственные значения для оператора Коши-Римана с нелокальными краевыми условиями при $\lambda \neq 2$, $\operatorname{Re} \lambda \neq 1$ для вещественной функции $u(z)=\operatorname{Re} \omega(z)$ на окружности $|z|=1$ редуцирована сначала к сингулярному интегральному уравнению, затем к линейному интегральному уравнению Фредгольма второго рода с непрерывным ядром

$$
\left(a^{2}(z)-b^{2}(z)\right) u(z)+\oint_{|t|=1} K(z, t) u(t) d t=0,
$$

где

$$
a(z)=\frac{(\lambda-2) e^{\lambda \bar{z}}+(\bar{\lambda}-2) e^{\bar{\lambda} z}}{2} ; \quad b(z)=\frac{(2-\lambda) e^{\lambda \bar{z}}-(2-\bar{\lambda}) e^{\bar{\lambda} z}}{2} ;
$$




$$
\begin{gathered}
K(z, t)=\frac{e^{\lambda \bar{t}}-e^{\lambda \bar{z}}}{t-z}\left\{\frac{\lambda a(z)}{\pi i}-\frac{\lambda b(z)}{\pi i}\right\}+\frac{e^{\bar{\lambda} t}-e^{\bar{\lambda} z}}{t-z}\left\{\frac{a(z)}{\pi i} \bar{\lambda}-\frac{2 b(z)}{\pi i}\right\}+ \\
+\frac{1}{t}\left\{\frac{(2+\lambda) b(z)\left(1-e^{\lambda \bar{z}}\right)}{2 \pi i}+\frac{a(z)+b(z)}{2 \pi i} \times\right. \\
\quad \times\left(e^{\lambda \bar{z}}-e^{\bar{\lambda} z}-\lambda-\bar{\lambda} e^{\bar{\lambda} z}+\frac{1}{2} \lambda e^{\lambda \bar{z}}+\frac{1}{2} \bar{\lambda} e^{\bar{\lambda} z}\right)+ \\
+\frac{i(a(z)+b(z))}{4 \pi(1-\operatorname{Re} \lambda)}(3 \lambda+3 \bar{\lambda})\left(e^{\bar{\lambda} z}-e^{\lambda \bar{z}}+\frac{1}{2} \bar{\lambda} e^{\bar{\lambda} z}-\frac{1}{2} \lambda e^{\lambda \bar{z}}+\lambda-\bar{\lambda}\right)- \\
\left.-\frac{(2+\lambda)(3 \lambda+3 \bar{\lambda})}{8 \pi(1-\operatorname{Re} \lambda)}\left(1-e^{\lambda \bar{z}}\right)\right\}+\frac{\bar{\lambda} e^{\bar{\lambda} t}}{t}\left\{\frac{(2+\lambda) b(z)}{4 \pi(1-\operatorname{Re} \lambda)}\left(1-e^{\lambda \bar{z}}\right)+\right. \\
\left.+\frac{a(z)+b(z)}{2 \pi(1-\operatorname{Re} \lambda)}\left(e^{\bar{\lambda} z}-e^{\lambda \bar{z}}+\frac{\bar{\lambda} e^{\bar{\lambda} z}}{2}-\frac{\lambda e^{\lambda \bar{z}}}{2}+\lambda-\bar{\lambda}\right)\right\}+ \\
+\frac{\lambda e^{\lambda \bar{t}}}{t} \frac{a(z)+b(z)}{2 \pi(1-\operatorname{Re} \lambda)} i\left(e^{\bar{\lambda} z}-e^{\lambda \bar{z}}+\frac{1}{2} \bar{\lambda} e^{\bar{\lambda} z}-\frac{1}{2} \lambda e^{\lambda \bar{z}}+\lambda-\bar{\lambda}\right) .
\end{gathered}
$$

Несколько замечаний о структуре ядра $K(z, t)$. Отметим, что

$$
a^{2}(z)-b^{2}(z) \neq 0 \quad \text { при }|z|=1 \text { и } \lambda \neq 2 .
$$

Итак, ядро $K(z, t)$ имеет представление

$$
\begin{aligned}
& K(z, t)=\frac{e^{\lambda \bar{t}}-e^{\lambda \bar{z}}}{t-z} A(z, \lambda)+\frac{e^{\bar{\lambda} t}-e^{\bar{\lambda} z}}{t-z} B(z, \bar{\lambda})+\frac{C(z, \lambda)}{t}+ \\
&+E(z, \lambda) D(t, \lambda)+F(z, \lambda) Q(t, \lambda),
\end{aligned}
$$

где $E, A, B, C, D, F, Q$ зависят от $t$ и $z$. Конкретный вид $E, A, B, C, D, F$, $Q$ можно восстановить из предыдущей формулы для $K(z, t)$. Заметим, что дробь $\left(e^{\bar{\lambda} t}-e^{\bar{\lambda} z}\right) /(t-z)$ с точностью до множителя $\exp (\bar{\lambda} z)$ зависит (в виде степенного ряда) от разности $(t-z)$. Аналогичное рассуждение верно и для $\left(e^{\lambda \bar{t}}-e^{\lambda \bar{z}}\right) /(t-z)$ при $|t|=|z|=1$, так как справедливо разложение

$$
\frac{e^{\lambda \bar{t}}-e^{\lambda \bar{z}}}{t-z} \frac{1}{t z}=-\frac{\exp (\lambda \bar{z})}{t z} \sum_{k=1}^{\infty} \frac{(\bar{t}-\bar{z})^{k-1}}{k !} \lambda^{k} .
$$

Последние три слагаемых в правой части (6) представляют сумму, соответствующую вырожденному ядру. Таким образом, ядро (6) состоит из вырожденной части, а другая часть содержит слагаемые, зависящие от разности. Вырожденные ядра изучались в монографии [18]. Интегральные операторы, зависящие от разности $(t-z)$, рассматривались в работах $[19,20]$. Однако прямое применение результатов этих работ не дает эффекта. Согласно результатам главы IV монографии [18] необходимым и достаточным условием существования ненулевого решения интегрального уравнения является обращение в нуль так называемого определителя Фредгольма. Определитель Фредгольма строится по формуле 


$$
\begin{aligned}
& \Delta(\lambda)=1+\frac{1}{1 !} \oint_{|z|=1} \hat{K}(z, z) d z+\frac{1}{2 !} \oint_{|z|=1} d z \oint_{|t|=1} d t\left|\begin{array}{cc}
\hat{K}(z, z) & \hat{K}(z, t) \\
\hat{K}(t, z) & \hat{K}(t, t)
\end{array}\right|+ \\
& +\frac{1}{3 !} \oint_{|z|=1} d z \oint_{|t|=1} d t \oint_{|\tau|=1} d \tau\left|\begin{array}{ccc}
\hat{K}(z, z) & \hat{K}(z, t) & \hat{K}(z, \tau) \\
\hat{K}(t, z) & \hat{K}(t, t) & \hat{K}(t, \tau) \\
\hat{K}(\tau, z) & \hat{K}(\tau, t) & \hat{K}(\tau, \tau)
\end{array}\right|+\ldots+,
\end{aligned}
$$

где $\hat{K}(z, t)=K(z, t) /\left(a^{2}(z)-b^{2}(z)\right)$.

Сходимость приведённого ряда следует из следующего неравенства Адамара. Если $D$ - определитель с комплексными элементами

$$
c_{h k}=p_{h k}+i s_{h k} \quad(h, k=1,2, \ldots, n),
$$

то верно неравенство

$$
D \leqslant P_{1} P_{2} \cdots P_{n}, \quad \text { где } \quad P_{h}=\left(\sum_{k=1}^{n}\left|c_{h k}\right|^{2}\right)^{1 / 2} .
$$

Действительно, при фиксированном $\lambda$, удовлетворяющем условиям $\lambda \neq 2$, $\operatorname{Re} \lambda \neq 1$, модуль ядра $|\hat{K}(z, t)|$ ограничен при $|t|=|z|=1$ некоторым числом $M$. Тогда из вышеуказанного неравенства Адамара следует, что определитель порядка $n$ с элементами, не превосходящими по модулю числа $M$, сам по модулю не больше $M^{n} n^{n / 2}$. Тогда $|\Delta(\lambda)|$ мажорируется следующим сходящимся рядом:

$$
1+2 \pi M+\frac{1}{2 !}(2 \pi)^{2} M^{2} 2+\frac{1}{3 !}(2 \pi)^{3} M^{3} 3^{3 / 2}+\ldots
$$

Таким образом, детерминант Фредгольма $\Delta(\lambda)$ определен при всех $\lambda$, удовлетворяющих условиям $\lambda \neq 2, \operatorname{Re} \lambda \neq 1$. Однако нахождение нулей определителя $\Delta(\lambda)$, записанного в вышеприведенной форме, неэффективно, поскольку $\Delta(\lambda)$ не является целой функцией от $\lambda$ и не выделена его главная часть.

В работе [17] вышеуказанным образом исследовалась структура ядра $K(z, t)$, при этом линейное интегральное уравнение Фредгольма (5) решить точно не удалось. Поэтому в настоящей работе для приближённого решения уравнения применим результаты работы [21], где дана оценка абсолютной величины разности между точным и приближенным решениями интегрального уравнения с выделением из данного ядра его главной части.

Введём функцию

$$
R(x, y)=K(x, y)-\tilde{K}(x, y)
$$

В нашем случае

$$
\begin{gathered}
R(z, t)=\frac{e^{\lambda \bar{t}}-e^{\lambda \bar{z}}}{t-z} \hat{A}(z, \lambda)+\frac{e^{\bar{\lambda} t}-e^{\bar{\lambda} z}}{t-z} \hat{B}(z, \bar{\lambda}), \\
\tilde{K}(z, t)=\frac{\hat{C}(z, \lambda)}{t}+\hat{E}(z, \lambda) D(t, \lambda)+\hat{P}(z, \lambda) Q(t, \lambda), \\
A(z)=e^{\lambda \bar{z}}\left(\frac{\lambda^{2}-2 \lambda}{2 \pi i}-\frac{2-\lambda}{\pi i}\right)+e^{\bar{\lambda} z}\left(\frac{\lambda \bar{\lambda}-2 \lambda}{2 \pi i}-\frac{2-\bar{\lambda}}{\pi i}\right),
\end{gathered}
$$


поэтому для модуля $A(z)$ справедлива оценка

$$
|A(z)|<e^{|\lambda|}\left(1+|\lambda|^{2}\right) .
$$

Точно так же оценивается функция $B(z)$ :

$$
|B(z)| \leqslant C\left(|\lambda|^{2}+1\right) e^{|\lambda|},
$$

где $C$ зависит от $\lambda$ и $z$,

$$
\begin{gathered}
B(z)=\frac{e^{\bar{\lambda} t}-e^{\bar{\lambda} z}}{t-z}\left\{\frac{a(z)}{\pi i} \bar{\lambda}-\frac{2 b(z)}{\pi i}\right\}, \\
a(z)=\frac{(\lambda-2) e^{\lambda \bar{z}}+(\bar{\lambda}-2) e^{\bar{\lambda} z}}{2}, \quad b(z)=\frac{(2-\lambda) e^{\lambda \bar{z}}-(2-\bar{\lambda}) e^{\bar{\lambda} z}}{2} .
\end{gathered}
$$

Таким образом, справедлива оценка для ядра возмущения

$$
|R(z, t, \lambda)| \leqslant C_{1} e^{4|\lambda|} \frac{|\lambda|^{2}+|\lambda|+1}{a^{2}\left(z_{0}(\lambda)\right)-b^{2}\left(z_{0}(\lambda)\right)},
$$

где $C_{1}$ не зависит от $\lambda, z, t$, т. е. в качестве малой величины можно взять правую часть последнего неравенства.

Далее оценим сверху резольвенту $|\tilde{\Gamma}(z, t ; \lambda)|$, соответствующую вырожденному ядру $\tilde{K}(z, t)$, оценивая снизу $\left|a^{2}(z)-b^{2}(z)\right|$. В то же время для величин $|C(z ; \lambda)|,|E(z ; \lambda)|,|D(t ; \lambda)|,|F(z ; \lambda)|,|Q(t ; \lambda)|$ нужны верхние неравенства. Поскольку величина $a^{2}(z)-b^{2}(z)$ при $\lambda \neq 2$ строго положительна, при фиксированном $\lambda$, для которого $\lambda \neq 2$, существует минимум по $z$, изменяющийся вдоль единичной окружности $|z|=1$ :

$$
\min _{|z|=1}\left(a^{2}(z)-b^{2}(z)\right)=a^{2}\left(z_{0}(\lambda)\right)-b^{2}\left(z_{0}(\lambda)\right)>0
$$

где

$$
\left|z_{0}(\lambda)\right|=1, \quad \tilde{\Gamma}_{1}(z, t)=\frac{(-1)}{\Delta_{1}(\lambda)}|\tilde{\Gamma}(z, t ; \lambda)|,
$$

то есть

$$
\tilde{\Gamma}_{1}(z, t)=\frac{(-1)}{\Delta_{1}(\lambda)} \Delta
$$

где

$$
\Delta=\left|\begin{array}{cccc}
\hat{C}(z, \lambda) & \hat{E}(z, \lambda) & \hat{F}(z, \lambda) & 0 \\
1+\oint \frac{\hat{C}(\tau, \lambda)}{\tau} d \tau & 1+\oint \frac{\hat{E}(z, \lambda)}{\tau} d \tau & 1+\oint \frac{\hat{F}(z, \lambda)}{\tau} d \tau & \frac{1}{t} \\
\oint \hat{C}(\tau, \lambda) D(\tau, \lambda) d \tau & \oint \hat{E}(z, \lambda) D(\tau, \lambda) d \tau & \oint \hat{F}(z, \lambda) D(\tau, \lambda) d \tau & D(t, \lambda) \\
\oint \hat{C}(\tau, \lambda) Q(\tau, \lambda) d \tau & \oint \hat{E}(z, \lambda) Q(\tau, \lambda) d \tau & \oint \hat{F}(z, \lambda) Q(\tau, \lambda) d \tau & Q(t, \lambda)
\end{array}\right|,
$$

$\Delta_{1}(\lambda)$ - соответствующий определитель Фредгольма, а все интегралы в $\Delta$ берутся по контуру $|\tau|=1$. 
Оценим сверху по отдельности величины, составляющие $R(z, t ; \lambda)$. Оценим

$$
\begin{gathered}
\frac{e^{\lambda \bar{t}}-e^{\lambda \bar{z}}}{t-z} \quad \text { при }|t|=|z|=1: \\
\left|\frac{e^{\lambda(\bar{t}-\bar{z})}-1}{t-z}\right| \cdot\left|e^{\lambda \bar{z}}\right| \leqslant e^{|\lambda|}\left\{|\lambda|+\frac{|\lambda|^{2} 2}{2 !}+\frac{|\lambda|^{3} 2^{2}}{3 !}+s\right\}=e^{|\lambda|} \frac{e^{2|\lambda|}-1}{2} \leqslant \frac{1}{2} e^{3|\lambda|} .
\end{gathered}
$$

Величины

$$
\max _{|z|=1}|C(z, \lambda)|, \max _{|z|=1}|E(z, \lambda)|, \max _{|t|=1}|D(t, \lambda)|, \max _{|z|=1}|F(z, \lambda)|, \max _{|t|=1}|Q(t, \lambda)|
$$

легко оцениваются мажорантой

$$
\frac{C}{|1-\operatorname{Re} \lambda|} e^{2|\lambda|}\left(|\lambda|^{2}+|\lambda|+1\right)
$$

Пусть при фиксированном $\lambda$ определитель Фредгольма $\Delta_{1}(\lambda) \neq 0$. Тогда, следуя [21], имеем верхнюю оценку для резольвенты $\tilde{\Gamma}_{1}(z, t ; \lambda)$ :

$$
\left|\tilde{\Gamma}_{1}(z, t ; \lambda)\right| \leqslant \frac{C_{1}\left(|\lambda|^{2}+|\lambda|+1\right)^{3} e^{6|\lambda|}}{\left|\Delta_{(\lambda)}\right|\left|a^{2}\left(z_{0}(\lambda)\right)-b^{2}\left(z_{0}(\lambda)\right)\right||1-\operatorname{Re} \lambda|^{3}}, \quad \operatorname{Re} \lambda \neq 1
$$

Учитывая все оценки, получим

$$
\begin{aligned}
2 \pi C_{1} e^{4|\lambda|}\left(|\lambda|^{2}+|\lambda|+1\right) \times & \\
& \times\left(1+2 \pi \frac{C_{2}\left(|\lambda|^{2}+|\lambda|+1\right)^{3} e^{6|\lambda|}}{\left|\Delta_{1}(\lambda)\right| \cdot\left|a^{2}\left(z_{0}(\lambda)\right)-b^{2}\left(z_{0}(\lambda)\right)\right| \cdot|1-\operatorname{Re} \lambda|^{3}}\right)<1 .
\end{aligned}
$$

Условие $\operatorname{Re} \lambda \neq 1$ может выполняться только если $C_{1}$ меньше единицы. В то же время на константу $C_{1}$ нельзя накладывать ограничения. Таким образом, из приведённых рассуждений видим, что применение результатов [21] требует малости величины, т. е. константа в нашем случае определяется малостью ядра возмущении $R(z, t)=K(z, t)-\tilde{K}(z, t)$.

Следовательно, при удачной аппроксимации ядра $K(z, t)$ вырожденным ядром $\tilde{K}(z, t)$ приходим к первому результату настоящей работы.

УтвеРЖДЕниЕ. Если $\lambda \neq 2$ и определитель Фредголъма вырожденного ядра $\tilde{K}(z, t)$ отличен от нуля, то $\lambda$ не является собственным значением исходной задачи (1)-(3) для уравнения Коши-Римана.

Для приближения ядра $K(z, t)$ вырожденным ядром $\tilde{K}(z, t)$ достаточно аппроксимировать величину $\left(e^{\bar{\lambda} t}-e^{\bar{\lambda} z}\right) /(t-z)$ вырожденным ядром. Поскольку

$$
\frac{e^{\bar{\lambda} t}-e^{\bar{\lambda} z}}{t-z}=e^{\bar{\lambda} z} \frac{e^{\bar{\lambda}(t-z)}-1}{t-z}=e^{\bar{\lambda} z}\left\{\bar{\lambda}+\frac{\bar{\lambda}^{2}(t-z)}{2 !}+\frac{\bar{\lambda}^{3}(t-z)^{2}}{3 !}+\ldots\right\}
$$


при $|\lambda|<R_{1}\left(R_{1}\right.$ - фиксировано) соответствующая аппроксимация выглядит так:

$$
e^{\bar{\lambda} z} U_{N}(t-z, \bar{\lambda}) \equiv e^{\bar{\lambda} z}\left\{\bar{\lambda}+\frac{\bar{\lambda}^{2}(t-z)}{2 !}+\ldots+\frac{\bar{\lambda}^{N}(t-z)^{N-1}}{N !}\right\},
$$

где $N$ - натуральное число (ниже будет указан алгоритм его выбора).

Таким образом, аппроксимирующее вырожденное ядро может быть записано в виде

$$
\begin{aligned}
\tilde{K}(z, t)=e^{\bar{\lambda} z}\left\{\bar{\lambda}+\frac{\bar{\lambda}^{2}(t-z)}{2 !}\right. & \left.+\cdots+\frac{\bar{\lambda}^{N}(t-z)^{N-1}}{N !}\right\} B(z, \bar{\lambda})- \\
-\frac{A(z, \lambda)}{t z} e^{\bar{\lambda} z}\{\lambda & \left.+\frac{\lambda^{2}(\bar{t}-\bar{z})}{2 !}+\cdots+\frac{\lambda^{N}(\bar{t}-\bar{z})^{N-1}}{N !}\right\}+ \\
& +\frac{1}{t} C(z, \lambda)+E(z, \lambda) D(t, \lambda)+F(z, \lambda) Q(t, \lambda),
\end{aligned}
$$

т. е. $\tilde{K}(z, t)$ записано через элементарные функции в явном виде. Для оценки погрешности аппроксимации $R(z, t)=K(z, t)-\tilde{K}(z, t)$ достаточно оценить погрешности приближений его отдельных слагаемых. В частности, пользуясь остаточным членом формулы Тейлора в форме Лагранжа, имеем оценки

$$
\begin{aligned}
\left|\frac{e^{\bar{\lambda} t}-e^{\bar{\lambda} z}}{t-z}-e^{\bar{\lambda} z} P_{N}(t-z, \bar{\lambda})\right| \equiv\left|e^{\bar{\lambda} z}\right| \frac{|\lambda|^{N+1}(t-z)^{N}}{(N+1) !}\left|e^{\theta(\lambda(t-z))}\right| & \leqslant \\
& \leqslant e^{3|\lambda|} \frac{|\lambda|^{N+1} 2^{N}}{(N+1) !} .
\end{aligned}
$$

Здесь учтено, что $|z|=|t|=1$. Точно так же оценивается величина

$$
\left|\frac{e^{\lambda \bar{t}}-e^{\lambda \bar{z}}}{t-z}-e^{\lambda \bar{z}} \tilde{P}_{N}(t-z, \bar{\lambda})\right| \leqslant e^{3|\lambda|} \frac{|\lambda|^{N+1} 2^{N}}{(N+1) !}
$$

Следовательно, для погрешности $|R(z, t)|$ справедливо неравенство

$$
\begin{aligned}
&|R(z, t)|<e^{3|\lambda|} \frac{|\lambda|^{N+1} 2^{N}}{(N+1) !}(|\hat{A}(z)|+|\hat{B}(z)|) \leqslant \\
& \leqslant C e^{4|\lambda|} \frac{|\lambda|^{N+1} 2^{N}\left(|\lambda|^{2}+1\right)}{(N+1) !} \frac{1}{a^{2}\left(z_{0}(\lambda)\right)-b^{2}\left(z_{0}(\lambda)\right)},
\end{aligned}
$$

где $C$ не зависит от $N$.

Оценим резольвенту, соответствующую вырожденному ядру $\tilde{K}(z, t)$. Резольвента $\tilde{\Gamma}_{N}$ имеет вид

$$
\tilde{\Gamma}_{N}=\frac{H_{N}(z, t, \lambda)}{\Delta_{N}(\lambda)}, \quad \Delta_{N}(\lambda)=\operatorname{det}\left|\delta_{i j}+\left\langle f_{j}, h_{j}\right\rangle\right|
$$

где $\Delta_{N}(\lambda)$ - определитель размерности $(2 N+3), H_{N}(\lambda)$ - определитель размерности $(2 N+4)$. 
Размерность $\Delta_{N}(\lambda)$ соответствует количеству слагаемых вырожденного ядра $\tilde{K}(z, t)$. Непосредственный подсчет позволяет $\tilde{K}(z, t)$ записать в виде

$$
\tilde{K}(z, t)=\sum_{j=1}^{2 N+3} f_{j}(z) h_{j}(t)
$$

где $\left\{f_{j}(z)\right\}$ и $\left\{h_{j}(t)\right\}$ представляют линейно независимые системы функции. В то же время определитель $H_{N}(z, t, \lambda)$ отличается от $\Delta_{N}(\lambda)$ одной строкой и одним столбцом, которые выглядят так:

$$
\left\|f_{1}(z), f_{2}(z), \ldots, f_{2 N+3}(z), 0\right\|
$$

последний столбец $H_{N}$ имеет вид

$$
\left\|0, h_{1}(t), h_{2}(t), \ldots, h_{2 N+3}(t)\right\|^{\top},
$$

где значок «Т» означает транспонирование.

Оценим сверху модуль определителя $H_{N}$. Для этого заметим, что при фиксированном $\lambda(\lambda \neq 2, \operatorname{Re} \lambda \neq 1)$ нормы каждой строки $H_{N}$ ограничены некоторой величиной $M$, не зависящей от натурального числа $N$. Тогда, согласно неравенству Адамара [18, стр. 192], имеем

$$
\left|H_{N}\right|<M^{2 N+4} \text {. }
$$

Подставляя правые части неравенства (9) и (10) в неравенство (7), получим соотношение для выбора натурального числа $N$ :

$$
C_{2} \frac{|\lambda|^{N+1} 2^{N}}{(N+1) !}\left(1+C_{3} \frac{M^{2 N+4}}{\left|\Delta_{N}(\lambda)\right|}\right)<1,
$$

где $C_{2}, C_{3}$ зависят от $\lambda$, но не зависят от $N$.

Поскольку $C^{N+2} /(N+1) ! \rightarrow 0$, ясно, что левая часть стремится к нулю при $N \rightarrow \infty$. Таким образом, выбор $N$ гарантируется. Сформулируем основной результат настоящей работы.

Теорема. Пусть $|\lambda|<R_{1}\left(R_{1}-\oint\right.$ фикировано) $u \lambda \neq 2, \operatorname{Re} \lambda \neq 1$. Пусть $N$ выбрано согласно (11). Определим $\tilde{K}(z, t)$ по формуле $(8)$, а через $\Delta_{N}(\lambda)$ обозначим определитель Фредгольма, соответствующий вырожденному ядру $\tilde{K}(z, t)$. Если $\Delta_{N}(\lambda) \neq 0$, то $\lambda$ является точкой резольвентного множества оператора Коши-Римана (1)-(3).

В теореме охарактеризованы те $\lambda$, при которых неоднородная краевая задача со смещением для оператора Коши-Римана (1)-(3) всюду разрешима в классе непрерывных в смысле Гёльдера функций на единичном круге, причём показана явная конструкция, аппроксимирующая решение неоднородной задачи. Для этого достаточно $u(z)$, определяемое как решение неоднородного линейного интегрального уравнения Фредгольма второго рода, аппроксимировать решениями неоднородного линейного интегрального уравнения с вырожденными ядрами. 


\section{СПИСОК ЛИТЕРАТУРЫ/ REFERENCES}

1. В. А. Стеклов, Основные задачи математической физики. Т. I, II, Петроград: Петроградский ун-т, 1922. [V. A. Steklov, Osnovnye zadachi matematicheskoj fiziki [Basic problems of mathematical physics], V.I, II, Petrograd, Petrogradskiy un-t, 1922 (In Russian)].

2. Ф. И. Франкль, "Обтекание профилей газом с местной сверхзвуковой зоной, оканчивающейся прямым скачком уплотнения" // Прикл. матем. и механика, 1956. Т. 20, № 2 . C. 196-202. [F. I. Frankl', "Subsonic flow past a profile with local supersonic zone bounded by a curved shock wave", Prikl. Matem. i Mekhanika, 1956, vol. 20, no. 2, pp. 196-202. (In Russian)]

3. А. М. Нахушев, "Новая краевая задача для одного вырождающегося гиперболического уравнения" // Докл. АН СССР, 1969. Т. 187, № 4. С. 736-739; A. M. Nakhushev, "A new boundary value problem for a degenerate hyperbolic equation", Sov. Math., Dokl., 1969, vol. 10, no. 4, pp. 935-938.

4. А. В. Бицадзе, А. А. Самарский, "О некоторых простейших обобщениях линейных эллиптических краевых задач" // Докл. АН СССР, 1969. Т. 185, №4. С. 739-740; A. V. Bitsadze, A. A. Samarskii, "On some simple generalizations of linear elliptic boundary problems", Sov. Math., Dokl., 1969, vol. 10, no. 4, pp. 398-400.

5. А. М. Нахушев, Задачи со смещением для уравнений в частных производных, М.: Наука, 2006. 287 с. [A. M. Nakhushev, Zadachi so smeshcheniyem dlya uravnenii $v$ chastnykh proizvodnykh [Problems with shifts for partial differential equations], Moscow, Nauka, 2006, 287 pp. (In Russian)]

6. Е. Ю. Арланова, “Задача со смещением для уравнения Бицадзе-Лыкова" // Вестн. Сам. гос. техн. ун-та. Сер. Физ.-мат. науки, 2012. №4(29). С. 26-36. doi: 10.14498/ vsgtu1103. [E. Yu. Arlanova, "The problem with shift for the Bitsadze-Lykov equation", Vestn. Samar. Gos. Tekhn. Univ. Ser. Fiz.-Mat. Nauki, 2012, no.4(29), pp. 26-36. (In Russian)].

7. А. М. Нахушев, "О современном состоянии краевых задач со смещением для основных типов уравнений в частных производных" / Трудъ Третъей Всероссийской научной конференици (29-31 мая 2006 г.). Часть 3, Дифференциальные уравнения и краевые задачи / Матем. моделирование и краев. задачи, Самара: СамГТУ, 2006. С. 170-173. [A. M. Nakhushev, "On the present state of boundary value problems with a shift for the main types of partial differential equations", Trudy Tret'yey Vserossiyskoy nauchnoy konferentsii [Proceedings of the Third All-Russian Scientific Conference] (29-31 May 2006). Part 3, Matem. Mod. Kraev. Zadachi, Samara, Samara State Technical Univ., 2006, pp. 170173. (In Russian)].

8. Д. Е. Касымова, А. Б. Тунгатаров, “Об одной краевой задаче со смещением для уравнения Карлемана-Векуа с сингулярной точкой" / Обобщенные аналитические функции u их приложения, Межвузовский сб. научн. трудов, Караганда: КарГУ, 1997. С. 4853. [D. E. Kasymova, A. B. Tungatarov, "On one boundary value problem with shift for Carleman-Vekua equation with a singular point", Obobshchennyye analiticheskiye funktsii $i$ ikh prilozheniya [Generalized analytic functions and their applications], Karaganda, Karaganda State Univ., 1997, pp. 48-53. (In Russian)].

9. М. Отелбаев, А. Н. Шыныбеков, "О корректных задачах типа Бицадзе-Самарского" // Докл. АН СCCP, 1982. Т. 265, №4. C. 815-819; M. Otelbaev, A. N. Shynybekov, "On well-posed problems of Bicadze-Samarskii type", Soviet Math. Dokl., 1982, vol. 26, no. 4, pp. 157-161.

10. Ying Wang, Yufeng Wang, "Two Boundary-Value Problems for the Cauchy-Riemann Equation in a Sector", Complex Analysis and Operator Theory, 2012, vol. 6, no. 6, pp. 11211138. doi: $10.1007 / \mathrm{s} 11785-010-0107-0$.

11. А. Ю. Тимофеев, "Краевая задача для обобщенного уравнения Коши-Римана в пространствах, описываемых модулем непрерывности" // Убимск. матем. журн., 2012. T. 4, № 1. C. 146-152. [A. Y. Timofeev, "Boundary problem for the generalized Cauchy- 
Riemann equation in spaces, described by the modulus of continuity", Ufimsk. Mat. Zh., 2012, vol. 4, no. 1, pp. 146-152. (In Russian)].

12. А. С. Ильчуков, "О поведении решения краевой задачи для обобщенного уравнения Коши-Римана"// Вестн. Удмуртск. ун-та. Матем. Мех. Компъют. науки, 2013. № 2. C. 27-34. [A. S. Il'chukov, "On behaviour of solution of boundary value problem for generalized Cauchy-Riemann equation", Vestn. Udmurtsk. Univ. Mat. Mekh. Komp. Nauki, 2013, no. 2, pp. 27-34. (In Russian)].

13. К. Н. Оспанов, М. Отелбаев, "Краевые задачи для обобщенной системы Коши-Римана с негладкими коэффициентами" // Докл. АН СССР, 1985. Т. 283, №1. С. 4649; K. N. Ospanov, M. Otelbaev, "Boundary value problems for the generalized CauchyRiemann system with nonsmooth coefficients", Soviet Math. Dokl., 1985, vol. 32, no. 1, pp. $40-42$.

14. К. Н. Оспанов, М. Отелбаев, "Об обобщенной системе Коши-Римана с негладкими коэффициентами" // Изв. вузов. Матем., 1989. №3. С. 48-56; K. N. Ospanov, M. Otelbaev, "A generalized Cauchy-Riemann system with nonsmooth coefficients", Soviet Math. (Iz. VUZ), 1989, vol. 33, no. 3, pp. 75-89.

15. В. А. Михайлец, Спектральные задачи с общими краевыми условиями: Диссерт. ... докт. физ.-мат. наук, Киев, 1989. [V. A. Mikhailets, Spektral'nyye zadachi s obshchimi krayevymi usloviyami [Spectral problems with general boundary conditions], Dissert. Doct. Phys. \& Math. Sci. Kiev, 1989 (In Russian)].

16. Д. К. Потапов, “Оценки дифференциального оператора в задачах со спектральным параметром для уравнений эллиптического типа с разрывными нелинейностями" // Вестн. Сам. гос. техн. ун-та. Сер. Физ.-мат. науки, 2010. №5(21). С. 268271. doi: 10.14498/vsgtu800. [D. K. Potapov, "Estimates of the DifferentialOperator in Problems with a Spectral Parameter for Elliptic-Type Equations with Discontinuous Nonlinearities", Vestn. Samar. Gos. Tekhn. Univ. Ser. Fiz.-Mat. Nauki, 2010, no. 5(21), pp. 268-271. (In Russian)].

17. Н. С. Иманбаев, Б. Е. Кангужин, Ж. Киргизбаев, "О фредгольмовости одной спектральной задачи, связанной с оператором Коши-Римана" / Bonросы устойчивости, прочности и управляемости динамических систем, Межвузовский сб. научн. трудов, М.: РГОТУПС, 2002. С. 54-59. [N. S. Imanbaev, B. E Kanguzhin, Zh. Kirgizbaev, "On the Fredholm property of one spectral problem related to Cauchy-Riemann operator", Voprosy ustoychivosti, prochnosti $i$ upravlyayemosti dinamicheskikh sistem [Questions of Stability, Durability and Controllability of the Dynamic System], Moscow, Russian State Open Technical Univ. of Railway Transport, 2002, pp. 54-59. (In Russian)].

18. Ф. Рисс, Б. Секефальви-Надь, Лекции по функциональному анализу, М.: Наука, 1979. 588 c.; F. Riesz, B. Sz.-Nagy, Functional analysis, New York, Frederick Ungar Publishing Co., 1955, xii+468 pp.

19. И. И. Кальмушевский, "О решениях некоторых интегральных уравнений с ядрами, зависящими от разности и суммы аргументов" // Дифферени. уравнения, 1980. Т. 16, № 5. C. 941-943. [I. I. Kal'mushevskiy, "On solutions of some integral equations with kernels depending on the difference and the sum of the arguments", Differents. Uravneniya, 1980, vol. 16, no. 5, pp. 941-943. (In Russian)].

20. Л. А. Сахнович, "О подобии операторов" // Сиб. матем. журнал, 1972. Т. 13, № 4. C. 868-883; L. A. Sakhnovich, "Similarity of operators", Siberian Math. J., 1972, vol. 13, no. 4, pp. 604-615. doi: 10.1007/BF00971053.

21. И. А. Акбергенов, "О приближенном решении интегрального уравнения Фредгольма и об определении его собственных значений" // Матем. сб., 1935. Т. 42, № 6. С. 679-698. [I. A. Akbergenov, "On approximate solution of the Fredholm integral equation and its eigenvalues", Mat. sb., 1935, vol. 42, no.6, pp. 679-698. (In Russian)].

Поступила в редакцию 29/IX/2013;

в окончательном варианте - 18/XI/2013;

принята в печать $-27 / \mathrm{I} / 2014$. 
MSC: 34L10, 34B09, 34B10

\section{EIGENVALUE PROBLEM FOR DIFFERENTIAL CAUCHY-RIEMANN OPERATOR WITH NONLOCAL BOUNDARY CONDITIONS}

\section{N. S. Imanbaev}

Kh. Yasavi International Kazakh-Turkish University,

2, pl. Esim-Khan, Turkestan, 487010, Kazakhstan.

We consider the reduced spectral problem for the Cauchy-Riemann operator with nonlocal boundary conditions to Fredholm linear integral equation of the second kind with a continuous kernel. The corresponding Fredholm determinant is defined for all spectral parameters, excepting the points: two and one. Finding zeros of the Fredholm determinant recorded in this form is inefficient, because it is not an entire function of the spectral parameter and the main part of the determinant is not separated. Moreover, we study the structure of the kernel by the method shown above, and establish that the linear Fredholm integral equation could not be solved exactly. Therefore, for its approximate solution the results of I. Akbergenov have been applied, where the estimates of the magnitude of the difference between the exact and approximate solutions of the integral equation are given, main part of the kernel is separated. In this case, the spectral parameters are described under which the nonhomogeneous boundary value problem with shift for the Cauchy-Riemann equations is solvable everywhere in the class of continuous functions on the unit circle. Moreover, the design of the approximated solution of the nonhomogeneous boundary value problem is given.

Keywords: Cauchy-Riemann operator, space of continuous functions, Fredholm property, resolvent set, problem with shift, kernel, Fredholm determinant.

Received 29/IX/2013;

received in revised form $18 / \mathrm{XI} / 2013$;

accepted $27 / \mathrm{I} / 2014$.

ISSN: 2310-7081 (online), 1991-8615 (print); doi: http://dx.doi.org/10.14498/vsgtu1264 (C) 2014 Samara State Technical University.

Citation: N. S. Imanbaev, "Eigenvalue Problem for Differential Cauchy-Riemann Operator with Nonlocal Boundary Conditions", Vestn. Samar. Gos. Tekhn. Univ., Ser. Fiz.-Mat. Nauki [J. Samara State Tech. Univ., Ser. Phys. \& Math. Sci.], 2014, no. 1(34), pp. 25-36. doi: 10.14498/vsgtu1264. (In Russian)

Author Details: Nurlan N. Imanbaev (Cand. Phys. \& Math. Sci.), Professor, Dept. of Mathematics.

E-mail address: imanbaevnur@mail.ru 